\title{
ZUR KENNTNIS JAVANISCHER AGROMYZINEN
}

VON

\author{
J. C. H. DE MEIJERE \\ Amsterdam.
}

(Mit 5 Figuren im Tekst)

\begin{abstract}
Ueber ostindische Agromyzinen ist bis jetzt nur wenig bekannt geworden. Ausser einigen älteren Angaben habe ich selbst in meinen „Studien über südostasiatische Dipteren” einige Arten beschrieben, welche im Verzeichnis zu den Studien (Studien XIV, Tijdschr. v. Entom. LX, 1918, p. 352) angegeben sind. Nur von Agromyza tephrosiae de Meij. konnte ich bis damals etwas über die Larve hinzufügen.

Im Jahre 1920 erhielt ich von Herrn P. VAN DER GOOT einige von ihm gezüchtete Arten, zu welchen später noch einiges Material von den Herren LEEFMANS und vaN HEURN hinzukam. Ich möchte hier etwas über diese Arten veröffentlichen als weiteren Schritt in dieses schwierige Gebiet, welche mich auch gerade deswegen interessiert, weil ich augenblicklich mit der biologischen Erforschung der europäischen Arten dieser Familie beschäftigt bin. Die hier zu erwähnenden Arten stammen alle aus Kulturpflanzen; von den Minenerzeugern in den wilden Gewächsen des malayischen Archipels ist kaum etwas bekannt.

Merkwürdigerweisé gehört die Mehrzahl der javanischen Arten der Gattung Melanagromyza Hendel an, welche in Europa gerade spärlich vertreten ist. Unter den gesammelten Larven finden sich sehr merkwürdige, welche Entwicklungsrichtungen zeigen, wie sie unter den europäischen bis jetzt noch nicht vertreten waren.

MALLOCH hat vor einigen Jahren mehrere Arten aus Formosa beschrieben aus SAUTER's Ausbeute $\left.{ }^{1}\right)$. Ueber die Biologie hat er leider nichts mitteilen können; die Imagines sind in dieser Familie oft schwer trennbar, ich glaube jedoch nicht, dass eine der unten aufgeführten in seinem Material vertreten ist.

Ganz wie bei den europäischen Arten ergibt sich auch hier, dass die Larven oft bessere Unterschiede aufweisen als die Imagines. Weil ich überdies die meisten der letzteren nur nach AlcoholExemplaren habe beschreiben können, so konnte ich nicht alle Merkmale mit genügender Sicherheit feststellen. Bei der grossen Zahl der noch zu erwartenden ähnlichen Arten verzichte ich einstweilen auf eine Tabelle, welche doch nur sehr fragmentarisch sein würde.
\end{abstract}

\section{MELANAGROMYZA PHASEOLI Coq.}

Syn. Agromyza destructor Malloch (Philippinen).

Java (v. D. Goot leg.); Medan (Sumatra, Corporaal leg.).

Dies ist eine kleine, schwarze, ins Schwarzblaue ziehende, mässig glänzende Art. Die Stirne ist mattschwarz, relativ lang und schmal, ungefähr so breit wie ein Auge, über den Fühlern mit schwacher

1) Malloch J. R. Formosan Agromyzidae. Ann. Mus. Nation. Hungar. XII, 1914, p. 306-336. 
Wölbung; charakteristisch ist das glänzend schwarze, lange und schmale Scheiteldreieck, welches sich bis etwas jenseits der Stirnmitte erstreckt. Periorbiten sehr schmal, mit nach oben und hinten gerichteten Härchen. Das kurze Untergesicht ist matt schwarzgrau, in der Mitte mit etwas erhabener Linie. Fühler schwarz, $3^{\text {tes }}$ Glied rund, Borste relativ lang, an der Wurzel etwas verdickt. Taster schwarz, Rüssel nicht verlängert, schwarzbraun mit etwas helleren Labellen. Thorax mit jederseits 2 Dorsocentralborsten. Beine schwarz, Flügel hyalin, Entfernung der beiden Queradern ungefähr so lang wie die hintere. Letzter Abschnitt der $5^{\text {ten }}$ Längsader kürzer als der vorletzte. Schüppchen schmutzigweiss, mit dunklen Wimpern. Schwinger schwarz. Flügellänge $1,5 \mathrm{~mm}$.

Larve von relativ langer, schmaler Gestalt. Der eine Mundhaken gross, schwarz, vorn mit einem starken Zahn und kleinem zweiten Zahn, der andere halb so lang, nur mit kleinerem Endzahn; dicht hinter den Mundhaken beiderseits eine schmale schwarze Gräte, welche sich nach oben erstreckt. Auf der Stirne ein fingerförmiger Anhang. Schlundgerüst schwarz, wenigstens im vorderen Teile, das Uebrige wenig gefärbt. Der unpaare Abschnitt relativ lang. Kopfabschnitt ohne Wärzchen.

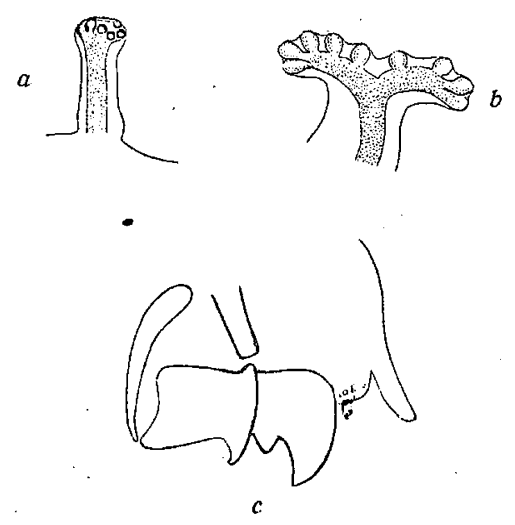

Fig. 1.

Melanagromyza phaseoli Coq. Larve.

$a$ Vorderstigma. $b$ Hinterstigma.

$c$ Vorderende des Körpers mit Stirn-

fortsatz und den 2 Mundhaken.

Die Warzengürtel ziemlich schmal und von schwacher Entwicklung; die Warzen stehen in deutlichen Querreihen, sind sehr klein, dreieckig, wenig gefärbt, namentlich vorn und hinten in den Gürteln sind 2-3 Reihen am deutlichsten, die Mitte des Gürtels bildet ein breiter Ring mit sehr wenigen- Wärzchen.

Vorderstigmen klein, kurz stabförmig vorragend, an der Spitze kaum verbreitert, mit ca. 6 Knospen in einem Bogen. Hinterstigmen dicht neben einander als kurze, konische Fortsätze vorspringend; je mit 2 fast gleichen Hörnern, zusammen mit ca. 10 Knospen. Hinterende etwas verschmälert, gerundet, nackt; zu beiden Seiten der Analöffnung ein kurzes Läppchen mit abgerundetem Rande.

Puparium braungelb, relativ schmal, mit wenig erkennbaren Ringgrenzen, überall fast gleich breit; Stigmen wenig vorragend.

Von VAN DER GOOT gezüchtet aus jungen Pflanzen von Phaseolus vulgaris, findet sich auch an Soja hispida, Vigna catjang, Phaseolus radiatus u.s. w., auf Java namentlich an Kedeleh (Soja hispida) und Phaseolus-Arten. Herr vaN DER GOOT hatte schon vorher einige Exemplare Herrn MALLOCH zugesandt, welche sie ihm als phaseoli Coq. bestimmte und die Synonymie mit destructor Mall. angab, welche also wohl gesichert ist. Auf diese Art bezieht sich auch dịe Mitteilung von J. B. CorporaAl in: Meded. $\mathrm{N}^{0}$. IX Alg. Ser. Alg. Proefstat. A. V. R. O. S., p. 8: „Jonge zaailingen van Kratok of "Limabeans” (Phaseolus lunatus L.) werden zoo goed als alle vernietigd door den aanval van een zeer klein vliegje”. Nach seiner brieflichen Mitteilung wurden von den eben gekeimten Pflänzchen die Stengel bisweilen von 10-20 Larven ausgehöhlt, so dass sie abstarben. Es ist auch dieselbe Art, über welche ich in Studien über südostasiatische Dipteren IX (Tijdschr. v. Entom. XLVII, 1914, p. 250) einige Angaben machte. Das Material stammte damals aus Banaran (Java; RoEPKE leg.), wo die Larven in Stengel und Wurzel europäischer Bohnen (Phaseolus) vorkamen.

\section{MELANAGROMYZA SOJAE Zehntn.}

Java (v. D. Goot leg.).

ZEHNTNER. De Kedeleh-boorder. De Indische Natuur I, 1900, p. 113.

Koningsberger. Ziekten van rijst enz. Meded. 's Lands Plantentuin LXIV, 1903, p. 87.

DAMMERMAN. Landbouwdierkunde van Oost-Indië, 1919, p. 81.

Sehr dunkel fast matt kupferfarbig, der Thorax mehr ins Purpurne, der Hinterleib ins Grüne, hinten ins Rötliche spielend.

Stirne relativ schmal, schmaler als ein Auge, mattschwarz, auch das Scheiteldreieck von dieser Farbe und dadurch kaum erkennbar. Fühler wie bei den vorigen Arten schwarz, $3^{\text {tes }}$ Glied rund, Borste 
kurz pubeszent. Untergesicht mässig kurz, schwarzgrau, in der Mitte ohne erhabene Linie, Backen sehr schmal. Taster schwarz, Labellen des Rüssels weiss.

Thorax mit 2 Dorsocentralborsten. Beine schwarz, Flügel glashell, das Geäder schwarzbraun; kleine Querader etwas, aber sehr wenig jenseits der Mitte der Discoidalzelle. Entfernung der Queradern etwas grösser als die gerade hintere Querader. Schüppchen weiss mit weisslichen Wimpern; Schwinger schwarz. Körper- und Flügellänge fast $2 \mathrm{~mm}$.

Larve schmal, gestreckt, ca. $3 \mathrm{~mm}$. lang, weiss. Der eine Mundhaken gross, schwarz, mit dicker, kurzer Spitze; der $2^{\text {te }}$ viel kleiner. Schlundgerüst schwarz, die Fortsätze beide kurz. Kein Stirnfortsatz vorhanden. Maxillarpalpen relativ gross, Kopfpapillen sehr deutlich.

Warzengürtel schmal, aus dreieckigen oder abgerundeten ziemlich kleinen Wärzchen bestehend, alle von fast gleicher Grösse, die meisten zerstreut, die vorderen, bisweilen auch die hinteren noch am meisten in Querreihen. Kopf ohne Wärzchen; Prothorakalgürtel breit, aus kleinen, in Reihen stehenden Zähnchen gebildet, ventral fehlend.

Vorderstigmen klein, knopfförmig, mit ca. 8 runden, sitzenden Knospen in 2 Reihen. Hinterstigmen aus einem Bogen von 7 Knospen bestehend, das Centrum lang vorragend, stabförmig, am Ende mehrfach ausgezackt und schwärzlich.

Hinterende abgestutzt, nackt.

Puparien braungelb, ziemlich schmal cylindrisch, die Hinterstigmen wenig vorragend, als 2 schwarze, von einander ungefähr um das Doppelte ihres Diameters getrennte Höckerchen. Vorderstigmen gleichfalls klein. Körper glatt, Ringgrenzen wenig deutlich.

Aus Larven gezüchtet, welche im Stengelmark von Kedeleh-Pflanzen (Soja hispida = Glycine soja) leben; dieselbe Fliege begibt sich auch an Phaseolus radiatus, Indigofera suffructicosa und Flemingia sp.

\section{MELANAGROMYZA DOLICHOSTIGMA n. sp.}

Java (v. D. Goot leg.).

Metallisch kupfergrün, Hinterleib bisweilen z. T. purpurn. Stirne mattschwarz, schmaler als ein Auge, mit kleinem, kupfergrünem Scheiteldreieck, welches so lang ist wie die Entfernung seiner vorderen Spitze von der Lunula; diese relativ gross, mattgrau. Härchen am Augenrande nach oben gerichtet. Fühler schwarz, Borste relativ lang, an der Wurzel etwas verdickt, sehr kurz pubeszent. Das kurze Untergesicht matt schwarzgrau, in der Mitte mit etwas erhabener Linie, welche in der oberen Hälfte etwas verdickt ist, ohne jedoch die Fühler von einander zu trennen. Taster schwarz, Rüssel nicht verlängert, Labellen heller.

Beine schwarz; Flügel glashell, das Geäder schwarzbraun bis schwarz; kleine Querader in der Mitte der Discoidalzelle; die Entfernung der Queradern deutlich länger als die gerade hintere Querader. Schüppchen schmutzig weiss, mit weissen Wimpern. Schwinger schwarz. Legeröhre kurz, schwarz. Körper- und Flügellänge 1,6 mm.

Larve lang und schmal. Mundhaken schwarz, ziemlich kurz, ungleich gross, je nur mit Endzahn. Schlundgerüst schwarz, kurz, die oberen Anhänge zweiteilig, der dorsale Flügel schmal, der ventrale viel breiter, nach hinten schmaler auslaufend; der ventrale Fortsatz wenig kürzer.

Warzengürtel deutlich, ziemlich breit, aus zerstreuten, dreieckigen, dunklen, spitzen Wärzchen gebildet, im Ganzen die im Inneren des Gürtels etwas grösser, die vorderen und hinteren öfters in Querreihen. Kòp ohne Wärzchen.
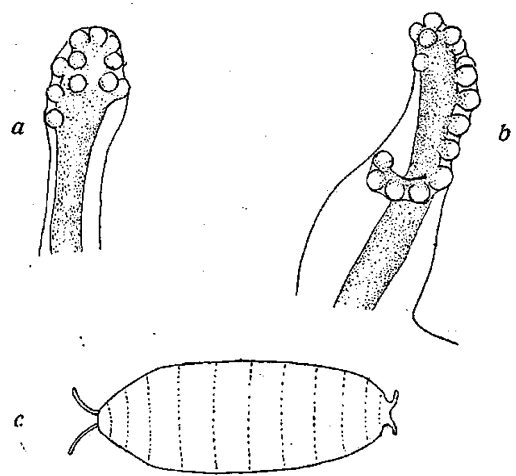

Fig. 3. Melanagromyza dolichostigma de Meij. Larve.

$a$ Vorderstigma. $b$ Hinterstigma $c$ Puparium.

Vorderstigmen lang stabförmig vorragend, gebogen; an der Spitze, welche etwas verdickt ist, findet sich an der Aussenseite das Stigma mit ca. 12 sitzenden Knospen. 
Hinterstigmen bédeutend kürzer vorragend, aber von ähnlicher Bildung, schmal dreieckige Fortsätze bildend, dicht neben einander entspringend, mit ca. 15 Knospen in 2 ungleichen Hörnern. Hinterende etwas verjüngt, nach vorn und hinten schief abgestutzt, ohne Wärzchen.

Puparium länglich oval, 2,5 mm. lang, gelbbraun, mit undeutlichen Ringgrenzen, durch die lang vorragenden Vorderstigmen leicht kenntlich; Hinterstigmen viel kürzer, mehr oder weniger schief nach aussen vorragend.

Aus den Zweigspitzen von Soja hispida, Phaseolus radiatus, Phaseolus calcaratus und Phaseolus vulgaris. Die Eier werden aussen an die Unterseite der noch nicht entfalteten oberen Blättchen abgelegt. Die Larven leben, mehrere zusammen, ausschliesslich im oberen Teil der Stengeln. Bisweilen sterben die Zweige $a b$, bisweilen wachsen sie durch und entstehen nur gallenähnliche Verdickungen des Stengels an der angegangenen Stelle. Die Fliege wird bisweilen ziemlich schädlich (VAN DER GooT).

In einer zweiten Tube hatte Herr vAN DER GOOT mir einiges Material (einige Puppen und ein paar Larven) dieser Art zugesandt mit der Angabe „aus den Schoten von Phaseolus radiatus”; ich konnte indessen keinen Unterschied mit den vorigen entdecken. Weil in dieser Tube auch das unten zu beschreibende Exemplar von $M$. decora vorhanden war, scheint mir die zuerst mitgeteilte Angabe über die Lebensweise einstweilen am zuverlässigsten.

\section{MELANAGROMYZA WEBERI n. sp.}

Java (VAN DER GOOT leg.).

Metallisch grüne Art. Stirn hinten ungefähr so breit wie ein Auge, mattschwarz, das Scheiteldreieck sehr gross, bis zur Lunula reichend, welche gleichfalls relativ gross und matt grauschwarz gefärbt ist. 4 Orbitalborsten vorhanden. Cilien am Augenrande gerade abstehend oder nach oben gerichtet. Fühler schwarz, das $3^{\text {te }}$ Glied rund; Borste schwarz, sehr kurz pubeszent. Taster schwarz, Labellen heller. Untergesicht sehr kurz, die nicht verlängerten Fühler dadurch beinahe den Mundrand erreichend. Thorax und Schildchen glänzend metallisch grün, Abdomen etwas mehr ins Rötliche ziehend; Legeröhre relativ lang, konisch, schwarz. Beine schwarz. Flügel glashell; das Geäder namentlich
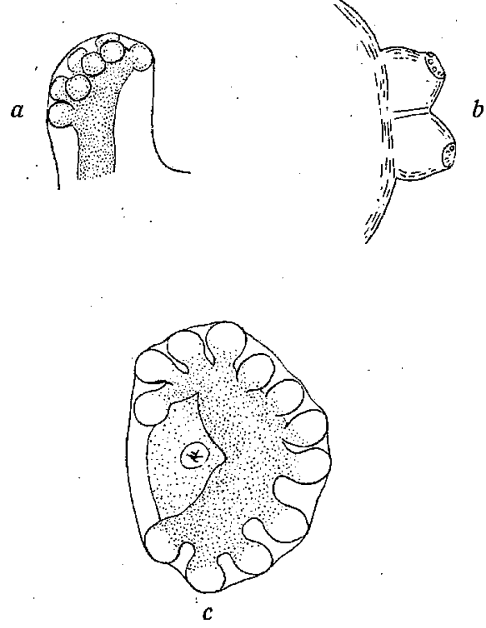

Fig. 4. Melanagromyza Weberi de Meij. Larve.

$a$ Vorderstigma. $b$ Hinterstigma.

$c$ Hinterende des Pupariums. an der Wurzel deutlich gelbbraun; kleine Querader in der Mitte der Discoidalzelle, hintere Querader etwas schief gestellt, Entfernung der Queradern deutlich grösser als die hintere. Schüppchen weiss, mit weissen Wimpern; Schwinger schwarz.

Körper- und Flügellänge $2,5 \mathrm{~mm}$.

Larve. Mundhaken schwarz, ungleich gross, nur mit Endzahn; Kopfabschnitt ohne Wärzchen. Schlundgerüst mit tief zerschlitzten oberen Fortsatzen und etwas kürzeren unteren, braungelb, der kurze unpaare Abschnitt nur ganz vorn schwärzlich. Warzengürtel aus dreieckigen, wenig gefärbten, ziemlich spitzen Wärzchen bestehend, welche alle von gleicher Grösse und zerstreut sind.

Vorderstigmen einhörnig, mit ca. 8 sitzenden Knospen. Hinterstigmen oval, flach, mit 2 gleichen Hörnern und 12 sitzenden Knospen. Hinterende abgestutzt-gerundet, nackt.

Puparium gelbbraun, glatt, die Wärzchen sehr klein und nicht gefärbt, die Gürtel mässig breit, an den Seiten sehr breit und aus zahlreichen Reihen bestehend, hier nur durch schmale, nackte Bänder getrennt. In den Zwischenräumen sind die Sinnespapillen als kleine, glänzende Punkte erkennbar. Hinterstigmen sehr kurz kegelförmig vorragend, dicht neben einander liegend.

Von Herrn VAN DER GOOT aus reifenden Schoten von Cajanus indicus und Flemingia sp. gezüchtet; die Larven leben im unreifen Saat.

Es sei mir erlaubt diese Art meinem hochverehrten Lehrer und Kollegen Herrn Prof. MAX WEBER, zu seinem siebzigsten Geburtstag zu widmen. 
Java (VAN DER Goot leg.), 1 Ex.

Stirne ungefähr so breit wie ein Auge, mattschwarz, das Scheiteldreieck sehr klein, sehr dunkel metallisch, fast bronzefarben, viel kürzer als seine Entfernung von der Lunula, diese ziemlich gross, weissgrau. Fühler schwarz.

Untergesicht mit etwas erhabener Mittellinie, Taster schwarz, Labellen etwas heller.

Thorax sehr dunkel metallischgrün bis bronzefarben, namentlich vorn dunkel. Hinterleib metallisch purpurn, die Vorderränder der Ringe metallisch blaugrün, der vorletzte Ring ins Kupfergelbe übergehend. Der Hinterleib ist ziemlich dicht und lang schwarz behaart, dadurch von matterem Aussehen als der Thorax. Beine schwarz. Flügel glashell, mit schwarzbraunem Geäder, die kleine Querader in der Mitte der Discoidalzelle, die hintere Querader etwas kürzer als die Entfernung der Queradern, gerade. Schüppchen weiss mit weissen Wimpern, Schwinger schwarz.

Körper- und Flügellänge $2 \mathrm{~mm}$.

Aus Schoten von Phaseolus radiatus, zusammen mit mehreren Puppen und Larven von M. dolichostigma de Meij.

\section{MELANAGROMYZA THEAE Green.}

Java: Rarahan, 1400 M.; Tjapoes, August (LeEfmAns leg.); die Larve miniert in Theeblättern.

Schwarz, der Thorax nur wenig, der Hinterleib stärker glänzend. Stirne mattschwarz, das Scheiteldreieck mässig glänzend, schmal bis zur Stirnmitte sich erstreckend; Periorbiten als schmale, glänzende Linien am Augenrande erkennbar; Lunula niedrig, dadurch breit, grau bestäubt. 2 nach oben gerichtete obere und 2 untere Orbitalborsten vorhanden; Härchen am Stirnrande nach oben gerichtet. Fühlerborste kurz pubeszent; $3^{\text {tes }}$ Fühlerglied rund. Taster schwarz, Labellen dunkelbraun. Thorax jederseits mit 2 Dorsocentralborsten. Flügel fast glashell; $2^{\text {te }}$ Längsader fast gerade; $3^{\text {te }}$ und $4^{\text {te }}$ schwach nach unten gebogen; die Costa erstreckt sich bis zur $4^{\text {ten }}$ Längsader. Entfernung der Queradern geringer als die Länge der hinteren Querader, letzter Abschnitt der $5^{\text {ten }}$ Längsader etwas kürzer als der vorletzte. Schüppchen graulich schwarz gerandet und gewimpert. Schwinger und Beine schwarz. Körper- und Flügellänge ca. 1,5 mm.

Wegen der biologischen Uebereinstimmung glaube ich nicht fehlzugehen, wenn ich diese Art als theae bestimme. „Oscinis" theae Bigot i. litt. wird erwähnt in einer Abhandlung: "The tea insects of India", in Indian Museum Notes III, 1896, $\mathrm{N}^{0} 4, \cdot$ p. 28; die Larve soll die Blätter der Theepflanze minieren und verpuppt sich am Ende des Ganges. Weitere Notizen gibt der Entdecker, E. E. GREEN, im "Ceylon Independent”, welcher mir nicht zugänglich war; nach GREEN wäre die. Fliege wahrscheinlich eine Agromyza, was mit meiner Ansicht stimmt; die Melanagromyzen wurden ja früher auch in diese Gattung untergebracht.

\section{MELANAGROMYZA RICINI n. sp.}

\section{Aus Ricinus-Früchten, Java (van HeURN leg.).}

Schwarz, mässig glänzend, Thorax und Hinterleib nur sehr schwach ins Bronzefarbene ziehend. Stirne mattschwarz, Scheiteldreieck sehr klein, bei weitem nicht bis zur Stirnmitte reichend, schwarz, wenig glänzend, Periorbiten schmal, etwas glänzend, Härchen am Augenrande nach oben gerichtet. Lunula nicht gross. Fühler schwarz, das $3^{\text {te }}$ Glied mässig gross, rund. Borste relativ lang, kurz pubeszent, an der Basis schwach verdickt. Augen gross, Backen schmal, Taster schwarz; Rüssel mit braungelben Labellen.

Thorax schwarz, etwas bronzefarben, mässig glänzend, jederseits mit 2 Dorsocentralborsten, die hintere länger, die vorhandenen Borsten relativ lang. Brustseiten schwarz, schwach glänzend. Hinterleib schwarz, gleichfalls etwas bronzefarben. Legeröhre kurz und dick, nicht länger als breit, schwarz. Flügel glashell, $2^{\text {te }}$ Längsader gerade, $3^{\text {te }}$ und $4^{\text {te }}$ fast parallel, kleine Querader in der Mitte der Discoidalzelle, letzter Abschnitt der $4^{\text {ten }}$ Längsader fast 3 mal so lang wie der vorletzte, letzter Abschnitt 
der $5^{\text {ten }}$ etwas länger àls die hintere Querader. Schwinger schwarz, Schüppchen schwarzgrau, schwarz gerandet und -gewimpert. Beine ganz schwarz; Vorderschienen hinten in der Mitte mit mässig langer Borste. Körper- und Flügellänge ca. $2 \mathrm{~mm}$.

Diese Art muss Agr. setigera Malloch aus Formosa ähnlich sehen, bei welcher aber das Scheiteldreieck undeutlich sein soll, der letzte Abschnitt der $4^{\text {ten }}$ Längsader ist relativ länger $(3 \%$ mal so lang wie der vorletzte), auch ist die Art grösser $\left(3-3 \frac{1}{2} \mathrm{~mm}\right.$.).

Larven weiss, bis ca. $4,5 \mathrm{~mm}$. lang, cylindrisch. Mundhaken schwarz, kurz und breit, ungleich gross, je nur mit Endzahn. Am Schlundgerüst ist der unpaare vordere Teil schwarz, die Fortsätze sind braun, ungefähr so lang wie der unpaare Teil, obere Fortsätze mässig breit, aus sehr schmalem, dunklerem, oberem, und breitem, hellerem, unterem Flügel bestehend, beide Flügel hinten einander nicht

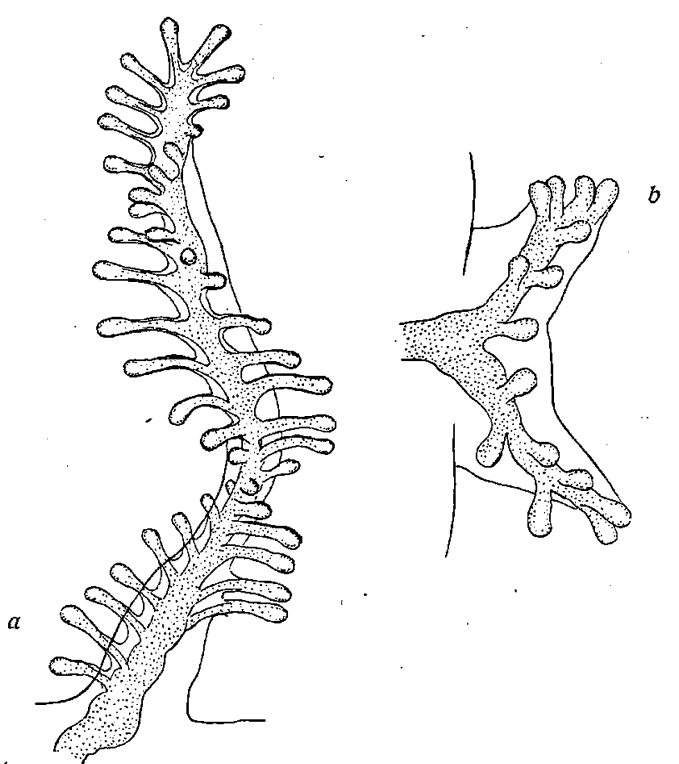

Fig. 5. Melanagromyza ricini de Meij. $a$ Vorderstigma. $b$ Hinterstigma. berührend. Vorderstigmen lang hornförmig vorragend, diese Hörner schwach S-förmig gebogen, mit einer grossen Anzahl auf ziemlich langen Stielen sitzender Knospen; jedes Stigma hat deren ca. 50, welche in 2 Spirallinien angeordnet sind, welche an der Spitze des Hornes in einander, übergehen.

Hinterstigmen zweihörnig, mit gleichen Hörnern und gleichfalls ziemlich lang gestielten Knospen, von welchen jedes Stigma ca. 15 aufweist.

Warzengürtel ziemlich schmal, aus ziemlich zahlreichen, meist zerstreut gelagerten, spitz dreieckigen, kleinen dunklen Wärzchen bestehend; die vorderen Reihen im ganzen etwas kleiner. Hinterende abgerundet, nackt.

Kopfabschnitt ohne Wärzchen und ohne Stirnfortsatz; Prothorakalgürtel dorsal gut entwickelt, aus in Querreihen und Quergruppen gelagerten dreieckigen Wärzchen gebildet.

Die Puparien sind gelb, von ovaler Gestalt, vorn etwas breiter als hinten. Durch die langen, schwarzen, divergierenden Vorderstigmen sind sie sehr auffällig. Nach

brieflicher Mitteilung von Jhr. VAN HEURN ist diese Art auf Java sehr schädlich für Ricinus, indem sie die Früchte dieser Pflanze zerstört; man findet die Larven (bzw. Puparien) oft zu 1,2 oder mehrere gleichzeitig in halbreifen und jüngeren Früchten eingebohrt.

Ueber die früher von mir, in meinen „Studien über südostasiatische Dipteren IV, IX und XIII”. beschriebenen Arten möchte ich noch folgende Bemerkungen hinzufügen:

\section{AGROMYZA ERYTHRINAE de Meij.}

Tijdschr. v. Entom. LIII, 1910, p. 160.

Gehört gleichfalls zu Melanagromyza. Die Stirne ist oben ungefähr so breit wie ein Auge, mattschwarz. Scheiteldreieck sehr dunkel metallisch grün, so lang wie seine Entfernung von der Lunula; diese mässig gross, grau. Stirnhärchen am Augenrande nach oben oder, meistens, nach hinten gerichtet. Flügelgeäder schwarzbraun, aber an der Wurzel deutlich heller, gelbweiss. Schüppchen weiss, mit weissen Wimpern.

\section{AGROMYZA PROVECTA de Meij.}

Tijdschr. v. Entom. LIII, 1910, p. 161.

Auch eine Melanagromyza. Härchen am Augenrande nach hinten gerichtet. Schüppchen dunkelbraun mit schwarzem Rand und Wimpern. 
AGROMYZA CORNUTA de Meij.

Tijdschr. v. Entom. LIII, 1910, p. 161.

Nach Hendel's Gattungstabelle eine Ophiomyia, aus der Nähe von Oph. curvipalpis Zett. Härchen der Stirnorbiten nach oben gerichtet. Lunula klein, seidenartig glänzend. Gesichtsmediankiel schmal, die Fühlerwurzeln trennend. Costa bis zur $4^{\text {ten }}$ Längsader reichend. Schüppchen weiss mit weissen Wimpern.

Das einzige Exemplar, ein $\sigma^{7}$, mit langen, aufgebogenen Vibrissenhörnchen. Vibrissenecke etwas vorgezogen. Von curvipalpis unterschiedet sich die Art durch die nicht schwarzgerandeten und -gewimperten Schüppchen.

\section{AGROMYZA ALBOHALTERATA de Meij.}

Tijdschr. v.' Entom. LVII, 1914, p. 249.

Ist eine Dizygomyza, welche carbonaria nahe steht. 4 Dorsocentralborsten jederseits vorhanden: auch die vordere gut entwickelt. Costa bis zur $4^{\text {ten }}$ Längsader reichend. Praescutellarborsten deutlich.

AGROMYZA TEPHROSIAE de Meij.

Tijdschr. v. Entom. LX, 1917, p. 249.

Scheint mir nach dem. Verhalten der Mediastina-Mündung gleichfalls eher zu Dizygomyza zu rechnen als zu Agromyza s. str., obgleich sie sich letzterer schon sehr nähert; der Endteil der Mediastina ist undeutlich und der Endteil der $1^{\text {ten }}$ Längsader nur wenig von der Costa getrennt. Praescutellarborsten vorhanden, vor der Quernaht keine Dorsocentralborsten. Gegen Agromyza sprechen auch die eigentümlichen Hinterstigmen der Larven, welche je dreiästig sind, jeder Ast mit drei Knospen an der Spitze.

An den Puparien ragen die Stigmen nicht vor, sie sind auch nicht verdunkelt, aber zeigen schon mit der Loupe die von der Larve beschriebene Dreiteiligkeit. Weiter nach unten zeigt das Hin-. terende einen feinen Querwulst; unter welchem als Längsspalte der Anus liegt. Am Hinterende der Larve finden sich sowohl unter den Hinterstigmen als in der Analgegend eine Anzahl spitzdreieckiger Wärzchen, am Kopfabschnitt kommen keine vor, der Prothorakalgürtel ist dorsal gut ausgebildet und besteht aus meistens in Querreihen gelagerten Wärzchen. Die Papillen ragen wenigstens in vorderen Körperabschnitt recht deutlich vor, so ist z. B. die unter dem Schlundgerüst ventral am Prothorax liegende Querreihe sehr deutlich. Das Schlundgerüst ist im unpaaren vorderen Abschnitt schwarzbraun, die Fortsätze sind viel heller braun, die oberen bestehen aus parallelem oberen und unteren Flügel, beide ziemlich schmal und fast von gleicher Länge, hinten fast in einander übergehend, der obere ist an der Wurzel breiter. Unterer Fortsatz mehr als halb so lang wie die oberen, am Ende jederseits mit grossem ovalen Loche. Wo in meiner Beschreibung der Larve von einem vierzähnigen Mundhaken die Rede ist, ist hierbei nicht mitgeteilt, dass es deren 2 gibt. Sie sind gleichgross, je mit 4 Zähnen versehen, von welchen der $1^{\text {te }}$.(Spitzenzahn), $3^{\text {te }}$ und $4^{\text {te }}$ mässig gross sind, der $2^{\text {te }}$ etwas grösser ist; die Zähne alternieren hier im Gegensatz zu den meisten anderen Agromyzinen kaum etwas.

Nach dem Obigen sind jetzt wenigstens von einigen ostindischen Arten auch die oft eigentümlichen Larven bekannt geworden. Es bleibt hier aber noch manches zu tun; von'Interesse wäre es z. B. auch die Larven von erythrinae und theae kennen $\mathrm{zu}$ lernen, letzteres um so mehr, als auch eine "Oscinis" coffeae von KonINGSBERGER beschrieben worden ist (Meded. 's Lands Plantentuin XX, 1897, pag. 25). 
Auch diese dürfte eine Agromyza s. l. sein, aber trotz mancher Anfrage ist es mir noch nicht gelungen, eine bezügliche Fliege, Larve oder Mine zu Gesicht zu bekommen. Desgleichen blieb mir die Phytomyza-sp., welche die Blätter des Zuckerrohrs minieren soll (van DevenTER, Handboek v. Suikerrietcultuur, II, p. 166) gänzlich unbekannt, und die Zahl der Agromyzinen der ostindischen Kulturpflanzen wird mit diesen wenigen Arten wohl nicht erschöpft sein. Dass auch trockene Larven in den Minen noch zur Untersuchung geeignet sind, dürfte die Zusendung des Materials erleichtern; zur vollständigen Kenntnis einer Art ist indessen die Zucht und Aufbewahrung einiger Stücke der verschiedenen Entwicklungsstadien empfehlenswert. 